the published literature and expert opinion. Costs were adjusted to 2016 prices.

Results There were 1,675 cases of adult AML between 1993 and 2013 with 733 (44\%) in women and 942 in men (56\%). There was a statistically significant annual percentage change of $2.45 \%$ in the incidence of AML in men while incidence in women also increased significantly by $1.21 \%$ per year...The costs associated with intensive chemotherapy management were $€ 89,750$ per case while the costs for transplantation, low-intensity chemotherapy and best supportive care were $€ 145,220$, $€ 11,790$ and $€ 12,745$ respectively. The annual cost of managing AML in Ireland between 2010 and 2015 was on average $€ 12.8$ million.

Conclusion The rising incidence of AML, together with improving survival means that more patients will be treated, achieve clinical remission and also require management for relapse. As novel treatments for this complex condition transition into practice, the costs of managing the disease will also rise. While routinely-collected cancer registration data can help to quantify this cost, better information on treatment patterns and recurrence will be necessary to accurately project and model the burden of this disease into the future.

\section{P77 UNMET NEEDS OF CANCER SURVIVORS IN IRELAND: A SCOPING REVIEW OF THE EXISTING EVIDENCE}

\author{
1,2M O'Connor*, 'B O'Donovan, 'C Donnelly. 'Research, The National Cancer Registry, \\ Cork, Ireland; ${ }^{2}$ School of Public Health, University College Cork, Cork, Ireland
}

10.1136/jech-2019-SSMabstracts.228

Background Due to advances in treatment and new drugs, more people in Ireland are surviving cancer. However, evidence is accumulating that cancer survivors have ongoing (and often unmet) needs for support and care. Supportive care interventions are required to meet these needs and maximise survivors' quality-of-life. International research illustrates the importance of targeting interventions to the specific needs and characteristics of the recipients. In order to address these variable needs, a better understanding of the unmet needs patients living with and beyond cancer is required. We conducted a scoping review of the current evidence on the unmet physical, emotional, practical and social needs of those living with and beyond cancer in Ireland.

Methods Five databases were searched to identify studies conducted in Ireland, published between 1998 and 2018. Studies reporting on adult survivors of childhood cancer, those including participants aged $\leq 17$ years, and those which reported on palliative care services were excluded. Unmet needs were classified into eleven primary domains including physical, psychosocial/emotional, family-related, social, practical, health system/ information and patient-clinician communication needs. A narrative synthesis of the data was undertaken.

Results 28 studies were included in the review consisting of both quantitative and qualitative studies. Most studies examined unmet needs between 1-3 yrs post treatment and the most frequently studied cancers were prostate, colorectal and breast cancer. Although melanoma, testicular and kidney cancers contribute significantly to total cancer prevalence, these received little attention in the Irish survivorship literature. Unmet physical, psychological and information needs were common across all cancer types with clear evidence of interrelationships between various unmet needs. This review also identified significant gaps in the literature in relation to a lack of availability of longitudinal data as well as a lack of information about the relationship between unmet needs and survivors' socio-demographic characteristics.

Conclusion As far as we are aware, this is the first scoping review of its kind conducted in Ireland. Gaps in the evidencebase for unmet needs, suggests that limited research has been conducted to understand the care needs of Irish cancer survivors. The planning and design of survivorship strategies in Ireland would benefit from routine collection of detailed information, with specific unmet need survey instruments, across multiple diseases. The National Cancer Strategy has identified survivorship care as a key challenge until 2026. The findings of this scoping review further reinforces the importance of enhancing survivorship services to address the unmet needs of cancer survivors.

\section{P78 PERINATAL MORTALITY IN IRELAND, 2016 - A NATIONAL CLINICAL AUDIT INTO PERINATAL MORTALITY IN THE REPUBLIC OF IRELAND}

${ }^{1}$ IB O'Farrell", ${ }^{1} E$ Manning, ${ }^{1} S$ Leitao, ${ }^{1,2} \mathrm{P}$ Corcoran, ${ }^{1} \mathrm{~J}$ McKernan, ${ }^{1} \mathrm{P}$ de Foubert, ${ }^{1}$ RA Greene. ${ }^{1}$ National Perinatal Epidemiological Centre, University College Cork, Cork, Ireland; ${ }^{2}$ National Suicide Research Foundation, University College Cork, Cork, Ireland

\subsection{6/jech-2019-SSMabstracts.229}

Background Perinatal mortality is a significant measurement of the outcome of obstetric and neonatal care. For this reason, in 2011, the National Perinatal Epidemiology Centre (NPEC) established the first national clinical audit of perinatal mortality in Ireland.

Methods Anonymised data on perinatal deaths that occurred between January 1 and 31 December 2016 were collected by contributors from each of the 19 maternity units in Ireland using a validated and standardised notification form. National rates per 1,000 births and corresponding 95\% confidence intervals were calculated. Customised birthweight centiles were calculated for all perinatal deaths using the Gestational Related Optimal Weight (GROW) software.

Results Based on the criteria of birthweight $\geq 500 \mathrm{~g}$ or gestation at delivery $\geq 24$ weeks, in total in 2016, 374 deaths were reported to the NPEC, of which 250 were stillbirths and 124 were early neonatal deaths. Stillbirth, early neonatal and perinatal mortality rates (PMR) were 3.9, 1.9 and 5.8 per 1,000 births respectively. The PMR corrected for congenital malformation was 3.6 per 1,000 births. Maternal factors such as advanced age and increased body mass index (BMI) were found to be associated with increased perinatal mortality. Major congenital anomaly was the primary cause of death in both stillbirths $(n=78 / 250,31.2 \%)$ and early neonatal deaths $(n=68 / 124,54.8 \%)$. The use of customised birthweight centiles showed that fetal growth restriction (FGR) was common. In cases of stillbirths, $60.0 \%$ of all stillbirths were classified as small for gestational age (SGA) ( $<10$ th customised birthweight centile) and $47.2 \%$ were severely SGA $(<3$ rd customised birthweight centile) compared to $33.9 \%$ and $25.0 \%$ of the cases of early neonatal deaths. Although the use of customised birthweight centiles showed that FGR occurred frequently, an antenatal diagnosis of FGR was only made in less than one in five $(19 \%, n=69$ of 363 , unknown for 11 cases) of perinatal deaths.

Discussion Clinical audit of perinatal outcomes in all maternity units in Ireland is vital for monitoring and improving patient 